\title{
Immunoproteomics approach revealed elevated autoantibody levels against ANXA1 in early stage gallbladder carcinoma
}

\author{
Javed Akhtar ${ }^{1,2}$, Ratna Priya ${ }^{1,2}$, Vaishali Jain ${ }^{1,3}$, Puja Sakhuja ${ }^{4}$, Anil Kumar Agarwal ${ }^{4}$, Surbhi Goyal ${ }^{4}$, \\ Ravindra Varma Polisetty ${ }^{5}$, Ravi Sirdeshmukh ${ }^{3,6}$, Sudeshna Kar ${ }^{2}$ and Poonam Gautam ${ }^{1 *}$ (D)
}

\begin{abstract}
Background: Early diagnosis is important for the timely treatment of gallbladder carcinoma (GBC) patients and may lead to increased survival outcomes. Here, we have applied serological proteome analysis (SERPA), an immunoproteomics approach, for the detection of 'tumor-associated antigens (TAAs) that elicit humoral response' in early stage GBC patients.

Methods: Total protein from pooled tumor tissue of GBC patients $(n=7)$ was resolved by two-dimensional gel electrophoresis (2-DE) followed by immunoblotting using pooled blood plasma from healthy volunteers $(n=11)$ or gallstone disease (GSD) cases $(n=11)$ or early stage GBC (Stage I and II) $(n=5)$ or GBC stage IIIA $(n=9)$. 2-D gel and immunoblot images were acquired and analyzed using PDQuest software to identify immunoreactive spots in GBC cases in comparison to controls. Proteins from immunoreactive spots were identified by liquid chromatographytandem mass spectrometric analysis (LC-MS/MS). Autoantibody levels for two of the functionally relevant proteins were investigated in individual plasma samples ( 52 cases and 89 controls) by dot blot assay using recombinant proteins.

Results: Image analysis using PDQuest software identified 25 protein spots with significantly high or specific immunoreactivity in GBC cases. Mass spectrometric analysis of 8 corresponding protein spots showing intense immunoreactivity (based on densitometric analysis) in early stage GBC or GBC stage IIIA cases led to the identification of 27 proteins. Some of the identified proteins include ANXA1, HSPD1, CA1, CA2, ALDOA and CTSD. Among the two proteins, namely ANXA1 and HSPD1 verified using a cohort of samples, significantly elevated autoantibody levels against ANXA1 were observed in early stage GBC cases in comparison to healthy volunteers or GSD cases (unpaired t-test, $p<0.05)$. Receiver operating characteristic (ROC) curve analysis for ANXA1 showed an Area under the Curve (AUC) of 0.69 , with $41.7 \%$ sensitivity against a specificity of $89.9 \%$ for early stage GBC. IHC analysis for ANXA1 protein showed 'high' expression levels in 72\% of GBC cases whereas all the controls showed 'low' expression levels.

(Continued on next page)
\end{abstract}

\footnotetext{
* Correspondence: gautam.poonam@gmail.com; poonamgautam.nip@gov.in

'Laboratory of Molecular Oncology, ICMR- National Institute of Pathology,

Safdarjung Hospital Campus, New Delhi 110029, India

Full list of author information is available at the end of the article
}

(c) The Author(s). 2020 Open Access This article is licensed under a Creative Commons Attribution 4.0 International License, which permits use, sharing, adaptation, distribution and reproduction in any medium or format, as long as you give appropriate credit to the original author(s) and the source, provide a link to the Creative Commons licence, and indicate if changes were made. The images or other third party material in this article are included in the article's Creative Commons licence, unless indicated otherwise in a credit line to the material. If material is not included in the article's Creative Commons licence and your intended use is not permitted by statutory regulation or exceeds the permitted use, you will need to obtain permission directly from the copyright holder. To view a copy of this licence, visit http://creativecommons.org/licenses/by/4.0/. The Creative Commons Public Domain Dedication waiver (http://creativecommons.org/publicdomain/zero/1.0/) applies to the data made available in this article, unless otherwise stated in a credit line to the data. 
(Continued from previous page)

Conclusions: The study suggests that the ANXA1 autoantibody levels against ANXA1 may be potentially employed for early stage detection of GBC patients. Other proteins could also be explored and verified in a large cohort of clinical samples.

Keywords: Autoantibody, Gallbladder carcinoma, Immunoproteomics, ANXA1, HSPD1

\section{Background}

Gallbladder carcinoma (GBC) is the fifth most common and aggressive malignancy of the gastrointestinal tract, with a high prevalence and incidence rate in Latin America (Chile) and Asian region (northern and northeast India) [1, 2]. Gallstone disease (GSD) is one of the major risk factors for GBC [3]. GBC is generally presented at the advanced stages due to its anatomic position and non-specificity of symptoms at early stages. Complete surgical resection is the potentially curative treatment for GBC at initial presentation with better survival outcomes, however, patients with metastatic GBC have a poor prognosis [4]. Five-year survival rate for early stages is higher (70-90\% in Stage I and $45-60 \%$ in Stage II, when treated with extended cholecystectomy) in comparison to advanced stage $\mathrm{GBC}$ patients $(\leq 20 \%)$ [5]. Therefore, the detection of GBC at an early stage may significantly improve the survival of these patients.

Antibodies against tumor-associated antigens (TAAs) have been reported in the serum of patients with various types of cancer and showed potential as biomarkers for early detection of cancer [6]. TAAs are generally present in low amounts at early stages and may not be detected by the available technologies; however, the autoantibodies generated against these TAAs are present in a high amount and may be detected in a pre-malignant stage. During the early stages of tumor development, these TAAs (proteins or peptides) may originate due to alterations at the genetic or protein levels and elicit humoral response [7]. As autoantibodies are highly stable in serum and are not proteolyzed, the detection of anti-TAA autoantibodies has the potential to improve assays for early detection of cancer [8].

Serological proteome analysis (SERPA), an immunoproteomics approach based on a classical proteomics workflow, is one of the most common tools that involve two-dimensional gel electrophoresis (2-DE), western blot and mass spectrometry analysis. The main advantage of using this method is the simultaneous analysis of whole protein, post-translational modifications and protein isoforms [9]. SERPA approach has been used for the identification of TAAs in several cancers including gingivo buccal complex cancer [10], hepatocellular carcinoma (HCC) [11], breast cancer [12]. Mustafa et al identified 18 and 9 immunoreactive spots, detected in atleast 4 out of 13 cholangiocarcinomas (CCA) sera, from the lysate of two $\mathrm{CC}$ cell lines and tumor tissue lysate respectively using this approach. They further validated anti-vimentin and anti-actin antibodies on colchicine-treated Hep2 cells and confirmed increased levels in $8(61 \%)$ and $3(23 \%)$ out of 13 CC cases respectively [13]. However, to the best of our knowledge, there is no report available on the identification of TAAs eliciting humoral response in GBC, till date.

The present study applies SERPA approach for detection of TAAs eliciting humoral response in GBC cases. Verification of two of the identified TAAs in a large cohort of clinical samples using Dot blot assay reveals ANXA1 as a candidate TAA to be considered further for clinical applications.

\section{Methods}

\section{Clinical samples}

Adult patients (with age $\geq 20$ years) diagnosed with GBC or GSD cases visiting Govind Ballabh Pant Institute of Postgraduate Medical Education and Research (GIPMER), New Delhi, were recruited for the study after approval from the Institutional Human Ethics Committee. Tumour Staging was done on the basis of clinical data of patients, histopathological evaluation and imaging tools, as per AJCC, 8th edition staging system [14]. Tissue samples from GBC cases $(n=7)$ and blood plasma samples from GBC cases $(n=52)$, GSD cases with no dysplasia $(n=48)$ and healthy volunteers $(n=41)$ were used in this study. Clinico-pathological data of these subjects are detailed in Table 1. Clinical parameters for the patients, wherever available $(\sim 70 \%)$, such as white cell count, liver enzymes (SGOT/SGPT/ALP) and cholestasis were collected.

Tissue samples were collected immediately after surgical resection from patients with GBC and stored at $80^{\circ} \mathrm{C}$ until used for further analysis. Peripheral blood (Approx. $5 \mathrm{ml}$ ) was collected in EDTA vials from patients with pre-operative GBC, pre-operative GSD and healthy volunteers. These blood samples were centrifuged at $2500 \times \mathrm{g}$ for $15 \mathrm{~min}$ at $4{ }^{\circ} \mathrm{C}$, clear plasma separated, aliquoted and stored at $-80^{\circ} \mathrm{C}$. All the samples were processed within 30 min of collection.

For in-house tissue microarrays (TMAs) preparation, formalin-fixed paraffin-embedded (FFPE) tissue samples from GBC cases and controls [Healthy individuals (gallbladder tissue from liver donors) and GSD cases] were drawn from GIPMER, New Delhi, India and National Liver Disease 
Table 1 Clinico-pathological data of case and control subjects. (A) Tissue samples and (B) Plasma samples used for the study

\begin{tabular}{|c|c|c|c|c|c|}
\hline Subjects & Total number & Number of males & Number of females & Mean age (Years) & Age range (years \\
\hline \multicolumn{6}{|l|}{ (A) Tissue samples } \\
\hline Total GBC Cases & 7 & 0 & 7 & 50.86 & $30-66$ \\
\hline \multicolumn{6}{|l|}{ Stages } \\
\hline GBC, Stage I & 1 & 0 & 1 & 38 & 38 \\
\hline GBC, Stage II & 1 & 0 & 1 & 65 & 65 \\
\hline GBC, Stage IIIA & 5 & 0 & 5 & 50.6 & $30-66$ \\
\hline \multicolumn{6}{|l|}{ Histological grade } \\
\hline Well-differentiated (G1) & 1 & 0 & 1 & - & - \\
\hline Moderately-differentiated (G2) & 4 & 0 & 4 & - & - \\
\hline Poorly-differentiated (G3) & 2 & 0 & 2 & - & - \\
\hline \multicolumn{6}{|l|}{ (B) Plasma samples } \\
\hline Total GBC Cases & 52 & 10 & 42 & 50.88 & $22-78$ \\
\hline \multicolumn{6}{|l|}{ Stages } \\
\hline GBC, Stage I & 6 & 1 & 5 & 39.66 & $22-47$ \\
\hline GBC, Stage II & 6 & 1 & 5 & 52.16 & $34-66$ \\
\hline GBC, Stage III & 17 & 2 & 15 & 52.76 & $30-66$ \\
\hline GBC, Stage IV & 23 & 6 & 17 & 52.08 & $38-78$ \\
\hline Early Stages (I and II) & 12 & 2 & 10 & 45.91 & $22-66$ \\
\hline Advanced stages (III and IV) & 40 & 8 & 32 & 52.37 & $30-78$ \\
\hline \multicolumn{6}{|l|}{ LN status } \\
\hline LN negative & 23 & 2 & 21 & 48.91 & $22-66$ \\
\hline LN positive & 29 & 8 & 21 & 52.44 & $38-78$ \\
\hline \multicolumn{6}{|l|}{ Histological grade } \\
\hline Well-differentiated (G1) & 5 & 0 & 5 & - & - \\
\hline Moderately-differentiated (G2) & 32 & 5 & 27 & - & - \\
\hline Poorly-differentiated (G3) & 15 & 2 & 13 & - & - \\
\hline Total Controls & 89 & 28 & 61 & 40.89 & $20-72$ \\
\hline GSD cases & 48 & 13 & 35 & 43.06 & $20-72$ \\
\hline Healthy group & 41 & 15 & 26 & 38.36 & $24-59$ \\
\hline
\end{tabular}

GBC Gallbladder carcinomas, GSD Gallstone disease, LN Lymph node

Biobank- Institute of Liver and Biliary Sciences (NLDBILBS), New Delhi, India, after approval from the Institutional Human Ethics Committee. Liver donors are donating healthy right lobe of the liver and the gallbladder is removed at the time of surgery. The details of the TMA preparation and samples used are described under 'Immunohistochemistry analysis' section.

\section{Protein extraction from GBC tissue samples}

Pooled tumor tissue $(50 \mathrm{mg}$ from seven GBC patients with Stage I, II and IIIA) was grinded in liquid nitrogen and added RIPA buffer $25 \mathrm{mM}$ Tris-Cl, $\mathrm{pH} 7.6+150$ $\mathrm{mM} \mathrm{NaCl}+2 \%$ (3-[(3-cholamidopropyl) dimethylammonio]-1-propanesulfonate (CHAPS)] with $1 \%$ protease inhibitor cocktail (Sigma, USA). The tissue homogenate was then sonicated (three bursts of $10 \mathrm{~s}$ each with $10 \mathrm{~s}$ of pause interval at $4{ }^{\circ} \mathrm{C}$ ) and centrifuged at $13,500 \times \mathrm{g}$ for $20 \mathrm{~min}$ at $4{ }^{\circ} \mathrm{C}$. The supernatant was separated and protein was quantified by Bradford assay. Sodium dodecyl sulphate- polyacrylamide gel electrophoresis (SDS-PAGE) was performed to analyze the protein profile of tissue lysate.

\section{Immunodepletion of GBC tissue lysate}

Tissue lysate was subjected to immunodepletion using Multiple Affinity Removal Spin Cartridge, human serum albumin (HSA)/immunoglobulin (Ig) G (Agilent Technologies, USA) to remove two of the abundant proteins (Albumin and IgG), as per the instructions by the manufacturer. Protein quantification followed by SDS-PAGE analysis was performed to confirm the removal of abundant proteins. 


\section{1-D immunoblot analysis}

For 1-D immunoblot analysis, immunodepleted GBC tissue proteins were separated by SDS-PAGE and electrotransferred to polyvinyl difluoride (PVDF) membrane. The blots were blocked with 5\% skimmed milk powder in TBST $[1 \times$ tris buffered saline $(10 \mathrm{mM}$ Tris-Cl, $\mathrm{pH} 7.4$ and $30 \mathrm{mM} \mathrm{NaCl}$ ) with $0.05 \%$ Tween 20 and $0.005 \%$ Triton-X-100] at RT for $1 \mathrm{~h}$. The blots were then incubated with pooled blood plasma from healthy individuals $(n=11)$ or GSD cases $(n=11)$ or GBC Stage I and II $(n=3)$ or GBC Stage IIIA $(n=9) \quad(1: 1000$ dilution $)$ at $4{ }^{\circ} \mathrm{C}$ overnight. The blots were then incubated with antihuman IgG conjugated with horseradish peroxidase (HRP) (1:40,000 dilution) (Thermo Scientific, USA) at RT for $1 \mathrm{~h}$ and were developed using the enhanced chemiluminescent (ECL) Kit (Millipore, USA). The images were acquired using Chemidoc MP imager and immunoblots were analyzed using Image Lab 4.1 software (Bio-Rad).

\section{2-D immunoblot analysis}

The immunodepleted GBC tissue proteins were separated using 2-DE method as described by Gorg et al [15]. Briefly, a total of $120 \mu \mathrm{g}$ protein which is dissolved in rehydration buffer [7 M Urea $+2 M$ Thiourea $+4 \%$ CHAPS $+1 \%$ dithiothreitol (DTT) $+1 \%$ N-Decanoyl-Nmethylglucamine (MEGA 10) with $0.2 \%$ bio-lyte with $\mathrm{pI}$ range $3-10$ and $0.002 \%$ bromophenol blue] was used for passive rehydration of immobilized-pH-gradient (IPG) strip, $11 \mathrm{~cm}, 3-10 \mathrm{NL}$ (Bio-Rad, USA). Proteins were separated in the first dimension on Protean 112 isoelectric focusing (IEF) system (Bio-Rad, USA) at $20^{\circ} \mathrm{C}$ using the following conditions: $250 \mathrm{~V}$ for $20 \mathrm{~min} ; 8000 \mathrm{~V}$ (gradual mode) for $1 \mathrm{~h} ; 8000 \mathrm{~V}$ (rapid mode) for an additional $26,000 \mathrm{Vh} ; 750 \mathrm{~V}$ (rapid) on hold with a maximum current of $50 \mu \mathrm{A} / \mathrm{IPG}$ strip. After IEF, the IPG strips were incubated in equilibration buffer [6 $\mathrm{M}$ urea, 30\% glycerol, $2 \%$ sodium dodecyl sulphate (SDS), $50 \mathrm{mM}$ Tris- $\mathrm{HCl}$ buffer, $\mathrm{pH} 8.8$ )] with $2 \%$ DTT for $15 \mathrm{~min}$ and then in equilibration buffer with $5 \%$ iodoacetamide for $15 \mathrm{~min}$ (Bio-Rad protocol). The proteins were further resolved in the second dimension by SDS-PAGE (4-20\% gradient midi gel) (Bio-Rad, USA) initially at $50 \mathrm{~V}$ for 30 min and then at $100 \mathrm{~V}$ until the dye front reached the bottom of the gel. The gel was stained with Coomassie Brilliant Blue R250 to visualize the proteins.

For 2-D immunoblot analysis, immunodepleted GBC tissue proteins $(120 \mu \mathrm{g})$ were separated in the first dimension by IEF (IPG strip $11 \mathrm{~cm}, 3-10 \mathrm{NL}$ ) and then in the second dimension by SDS-PAGE (4-20\% gradient gel) as described above. The proteins were then electrotransferred to the PVDF membrane using a semi-dry method (Bio-Rad). The blots were blocked with skimmed milk powder followed by incubation with pooled blood plasma from healthy individuals $(n=11)$ or GSD cases $(n=11)$ or GBC Stage I and II $(n=5)$ or GBC Stage IIIA $(n=9)$ (1:1000 dilution). The blots were then incubated with anti-human IgG conjugated with HRP (Thermo) (1:40,000 dilution) and were developed using ECL Kit (Millipore). All other conditions were the same as that of 1-D immunoblot analysis. The images were acquired using the ChemiDoc MP imaging system (Bio-Rad) and immunoblots were analyzed to identify protein spots showing immunoreactivity in GBC cases.

\section{In-gel digestion}

The 2-D immunoblot images were compared with 2-D gel images (Coomassie Stained) to identify corresponding immunoreactive protein spots on the 2-D gel using PDQuest software version 8.1. The coomassie-stained protein spots of interest were excised using glass capillaries (internal diameter $\sim 1 \mathrm{~mm}$ ) and incubated with $400 \mu \mathrm{l}$ of destaining solution $\left[25 \mathrm{mM} \mathrm{NH} \mathrm{NCO}_{3}\right.$ in $50 \%$ acetonitrile $(\mathrm{ACN})]$ in microfuge tube at RT. The destaining solution was changed after every $15 \mathrm{~min}$ till the gel pieces were completely destained followed by the addition of $400 \mu \mathrm{l}$ of $100 \% \mathrm{ACN}$ and incubated for 5 min. ACN was removed and dried the gel using a speed vacuum concentrator followed by the addition of $15 \mu \mathrm{l}$ of $10 \mathrm{ng} / \mu \mathrm{l}$ trypsin (prepared in $25 \mathrm{mM} \mathrm{NH}_{4} \mathrm{HCO}_{3}$ ) to the dehydrated gel and incubated overnight at $37^{\circ} \mathrm{C}$. The next day, $50 \mu \mathrm{l}$ of peptide extraction buffer $[0.3 \%$ Trifluoroacetic acid (TFA) in 50\% ACN] was added to each tube and incubated for $30 \mathrm{~min}$ with gentle shaking. The solution was then removed and stored in a fresh microfuge tube. This step was repeated twice and the solution was pooled with the previous tube and lyophilized to get trypsin digested peptides.

\section{Mass spectrometric analysis}

Tryptic digests from different protein spots were subjected to mass spectrometric analysis (nanoRPLC-MS/ MS) for protein identification. Nanoflow electrospray ionization tandem mass spectrometric analyses of peptide samples were carried out using Orbitrap QExactive plus (Thermo Scientific, Bremen, Germany) coupled with RS nano high-performance liquid chromatography (HPLC) 3000 system (Thermo Scientific, Bremen, Germany). Peptide samples were enriched using a C18 Trap column and separated on an Acclaim ${ }^{\text {Tw }}$ PepMap $^{\text {Tw }}$ 100 C18 analytical column $(3 \mu \mathrm{m}, 150 \mathrm{~mm}, 0.075 \mathrm{~mm}$ I.D.) at a flow rate of $300 \mathrm{nl} / \mathrm{min}$ using a linear gradient of $7-30 \%$ ACN over $65 \mathrm{~min}$. The Mass spectrometric analysis was carried out in a data-dependent manner with full scans acquired using the Orbitrap mass analyzer at a mass resolution of 70,000 at m/z 200. From each MS scan, 20 most intense precursor ions were 
selected for MS/MS fragmentation and detected at a mass resolution of 35,000 at $\mathrm{m} / \mathrm{z} 200$. The fragmentation was carried out using higher-energy collision dissociation (HCD) with $30 \%$ normalized collision energy. The ions selected for fragmentation were excluded for $30 \mathrm{~s}$. The automatic gain control for full FT-MS was set to 1 million ions and FT-MS/MS was set to 0.1 million ions with a maximum time of accumulation of $500 \mathrm{~ms}$. For accurate mass measurements, the lock mass option was enabled.

\section{Data analysis}

Protein identifications were carried out as follows. The MS data were analyzed using Proteome Discoverer (Thermo Fisher Scientific, Version 1.4). MS/MS search was carried out using the SEQUEST search engine against the NCBI RefSeq database version 81. Search parameters included trypsin as an enzyme with 1 missed cleavage allowed; precursor and fragment mass tolerance were set to $20 \mathrm{ppm}$ and $0.1 \mathrm{Da}$, respectively; Methionine oxidation was set as a dynamic modification while S-carboamidomethyl modification at cysteine was set as static modifications. The false discovery rate (FDR) was calculated by enabling the peptide sequence analysis using a decoy database. High confidence peptide identifications were obtained by setting a target FDR threshold of $1 \%$ at the peptide. For the identification of peptides/ proteins, signal to noise ratio applied was 1.5 or more and this is within the acceptable standards for the instrumentation platform used. Contaminant peaks such as peaks of keratin proteins were selected under the exclusion list. Proteins identified by $\geq 2$ unique peptides were considered as high confidence identifications. Molecular functions and localization of the identified proteins were derived from the human protein reference database, http://www.hprd.org, [16] and UniProt database, www.uniprot.org/uniprot.

\section{Dot blot assay}

Dot blot assay was performed for Annexin A1 (ANXA1) and heat shock protein 60 (HSPD1) to analyze the autoantibody levels in individual plasma samples. A total of $75 \mathrm{ng}$ recombinant human ANXA1 protein or HSPD1 (Abcam, USA) was spotted on the PVDF membrane. The blots were blocked with $5 \%$ skimmed milk powder in $1 \times$ TBST followed by incubation with individual blood plasma from healthy individuals $(n=41)$, GSD cases $(n=48)$, GBC Stage I and II $(n=12)$, GBC Stage IIIA $(n=11)$, GBC Stage IIIB $(n=6)$ and GBC Stage IVB $(n=23)$ (dilution 1:1000). The blots were then incubated with secondary antibody (anti-human IgG HRP conjugated) (1:40,000 dilution) followed by development using SuperSignal ${ }^{\mathrm{Tm}}$ West Femto Maximum Sensitivity Substrate (Thermo, USA). All other conditions were the same as that of 1-D immunoblot analysis. The images were acquired using Chemidoc MP (Bio-Rad, USA) and densitometric analysis was performed using Image Lab software version 4.1 (Bio-Rad, USA) to compare the autoantibody levels in controls and cases. Two of the plasma samples were included in all the dot blot experiments and were used for normalization.

\section{Statistical analysis}

Statistical analysis was performed using GraphPad Prism 5 . The autoantibody levels, indicated by density (arbitrary units) of immunoreactive spots of individual controls and cases were used for the analysis. Differences in autoantibody levels between two independent groups were tested with unpaired t-test (two-tailed) with confidence intervals of $95 \%$ and $p$-value of less than 0.05 was used to indicate statistical significance. The receiver operating characteristic (ROC) analysis for autoantibody levels against ANXA1 for various groups of GBC [Early stage (GBC stage I and II) vs healthy or GSD; Stage IIIA vs healthy or GSD; stage IIIB vs healthy or GSD; stage IVB vs healthy or GSD; early stage vs all controls; advanced stage (stage III and IV) vs all controls; LN negative GBC vs all controls; LN positive vs all controls] was performed leading to the estimates of the area under the curve (AUC) with 95\% confidence interval (CI) along with sensitivity and specificity. The autoantibody levels below the cut-off value were considered 'low' and above the cut-off value was considered as 'high'.

\section{Immunohistochemistry analysis}

IHC was performed on FFPE tissues using tissue microarray (TMAs) and individual tissue sections to analyze the expression of ANXA1 protein. In-house TMAs were prepared as follows. Two TMA blocks were constructed using the FFPE blocks and included 14 controls (2 healthy liver donors and 12 GSD cases) and 31 GBC cases (9 early stage and 22 advanced stage). Each TMA block consisted of 22 cores of $2 \mathrm{~mm}$ diameter, and Hematoxylin and Eosin (H \& E) - stained sections of the blocks were used to define tumor regions. Further, $4 \mu \mathrm{m}$ sections were cut from the TMA block for carrying out IHC. Individual tissue sections (FFPE) of GBC (7 early stage and 5 advanced stage) were also used for IHC analysis. In brief, after deparaffinization and rehydration of FFPE tissue sections, antigen retrieval was performed by immersing the slide in antigen retrieval buffer $(20$ $\mathrm{mM}$ Tris buffer, $\mathrm{pH}$ 9.0) at $90^{\circ} \mathrm{C}$ for $20 \mathrm{~min}$. Endogenous peroxidases were blocked with $0.03 \%$ hydrogen peroxide, and nonspecific binding was blocked with protein blocking reagent. Sections were then incubated for $1 \mathrm{~h}$ at RT with primary antibody against ANXA1 (dilution 1: 
4000, Cat. No. ab214486, Abcam, USA) followed by incubation with PolyExcel PolyHRP for $40 \mathrm{~min}$ at RT. Tissue sections were then incubated with Stunn DAB working solution for $5 \mathrm{~min}$ at RT (PathnSitu Biotechnologies, USA). Sections were counter stained with Mayer's hematoxylin, dehydrated and images were taken under the microscope. The distribution of staining and staining intensity across the section was observed under the microscope. Scoring criteria were based on both staining intensities and distributions. The staining intensity of cancer cells scored as $0,1+, 2+/ 3+$ indicating negative, low, and strong staining respectively. The distribution of staining of cancer cells was scored as 0 $(<10 \%$ of cells staining), $1+(10-<25 \%$ of cell staining), $2+(25-<50 \%$ of cells staining $)$ and $3+(\geq 50 \%$ of cells staining). ANXA1 expression was considered 'high' if the percentage distribution was $\geq 25 \%$ and 'low' if it was $<25 \%$. IHC data analysis was analyzed by two independent pathologists.

\section{Results}

In the present study, we have used immunoproteomics approach for the detection of TAAs, eliciting a humoral response in GBC patients, followed by clinical verification by dot blot assay using recombinant proteins. The overall workflow of the study is shown in Fig. 1.

\section{Clinical parameters}

The GBC samples $(n=52)$ used for the study included $23 \%$ early stage GBC cases $(n=12)$ and $\sim 80 \%$ female patients $(n=42)$ as shown in the demographic data. A

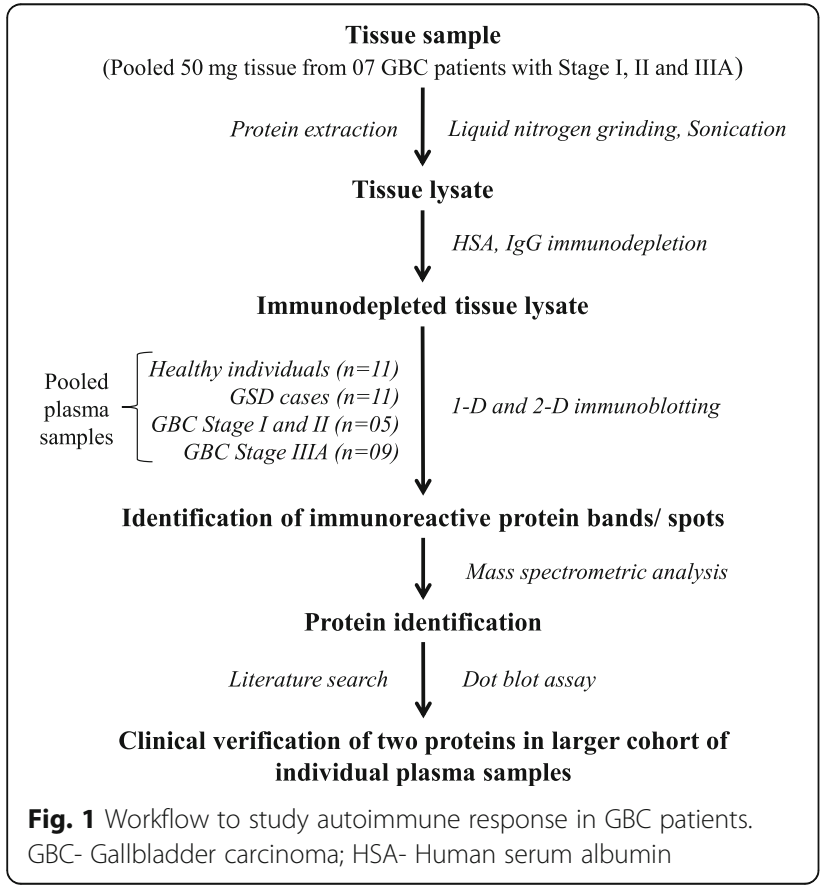

total of 23 cases were lymph node (LN) negative and 29 were LN positive (Table 1a). Among the GBC cases used for pooling of tissue samples, we find variations among the samples irrespective of their stage. There was no significant variations among the GSD and GBC early stage (stage I and II) used for pooling of plasma, however, there were variations among the GBC stage IIIA cases (Supplementary Figure S1). Analysis of the clinical parameters in GSD and GBC cases used for verifications (individual plasma) showed variations in clinical parameters among both the groups (Supplementary Figure S1).

\section{Autoantibody response analysis in GBC}

The 1-D Immunoblot analysis with immunodepleted GBC tissue lysate probed with pooled plasma from healthy individuals or GSD or early stage GBC (stage I and II) or GBC Stage IIIA showed 03 protein bands $(37 \mathrm{kDa}, 30 \mathrm{kDa}$ and $28 \mathrm{kDa})$ with high or specific immunoreactivity in early stage GBC, and 02 protein bands $(54 \mathrm{kDa}$ and $39 \mathrm{kDa})$ with high or specific immunoreactivity in GBC Stage IIIA (Fig. 2a, Supplementary Figure S3A). Further, 2-D immunoblot analysis using the same pool of plasma samples as above showed significantly high or specific reactivity for a total of 25 protein spots in GBC cases. These spots are marked with an arrow in the Coomassie-stained 2-D gel and 2-D immunoblots (see Fig. 2b and c, Supplementary Figure S3B). A total of 13 protein spots were found particularly in early stage and 10 protein spots in Stage IIIA, and 2 were common (spot no. 9 and 11) among both the groups.

\section{Protein identification}

Liquid chromatography-tandem mass spectrometric analysis (LC-MS/MS) of the four most intense immunoreactive protein spots (based on the densitometric analysis) in early stage GBC (Spot No. 9-12) or in GBC Stage IIIA (Spot No. 17-20), led to the identification of 17 and 12 proteins respectively (Fig. 2, Supplementary Table S1). The list of identified proteins and corresponding peptides is shown in Supplementary Table S2. We performed a literature search to assess the significance of the individual proteins for further clinical verifications. The 'molecular functions' of these proteins mainly include catalytic activity and transporter activity whereas the 'biological processes' include energy metabolism and transport. These proteins were majorly localized to the cytoplasmic, plasma membrane, extracellular (Supplementary Table S3). Some of the important proteins identified from immunoreactive spots in early stage GBC include carbonic anhydrase isoform 1 and 2 (CA1 and CA2), ANXA1, HSPD1, aldolase A and B (ALDOA and ALDOB), cathepsin D preproprotein 


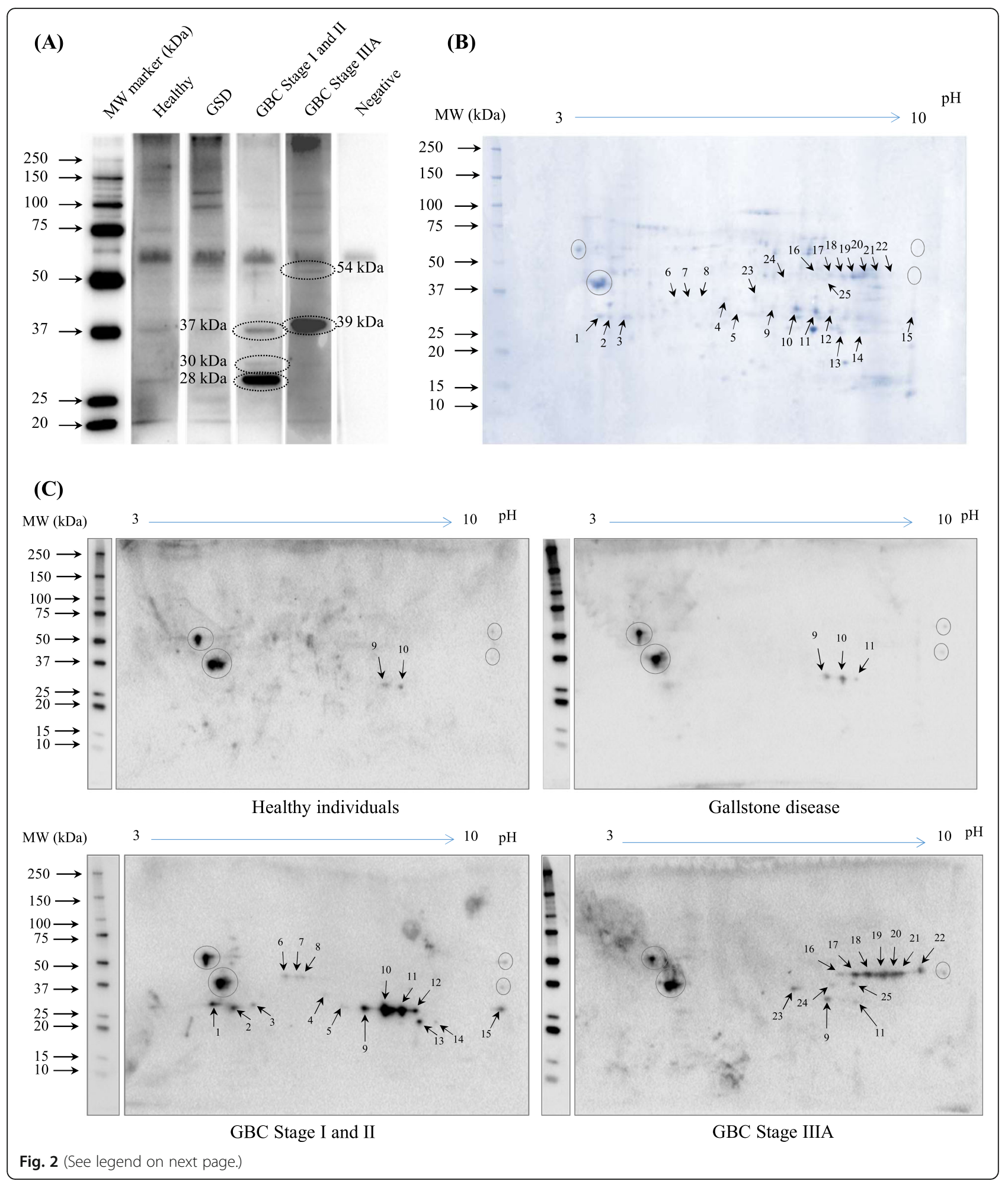




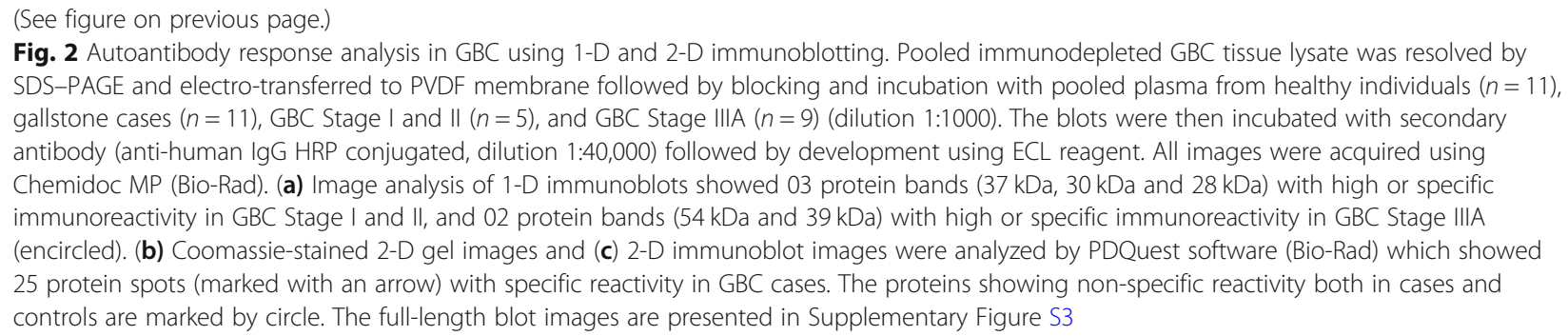

(CTSD) and plectin isoform 1d (PLEC) and those identified from immunoreactive spots in GBC Stage IIIA include ALDOA, ALDOB, arginase 1 (ARG1) and lactotransferrin isoform 2 (LTF). Two of the proteins, ALDOA and ALDOB were common among both the groups. Identification of multiple proteins from the individual gel spots could be presumably due to lower resolution of proteins through broad $\mathrm{pH}$ range used for iso-electric focusing in 2-DE.

Similarly, we also observed some individual proteins detected in multiple spots. For examples, among early stage $\mathrm{GBC}$, five proteins [ALDOB, CA1, hemoglobin subunit beta (HBB), immunoglobulin lambda-like polypeptide 5 isoform 1 (IGLL5), PLEC] were identified in $\geq 3$ protein spots, while five proteins [ANXA1, CA2, CTSD, delta (3,5)-Delta (2,4)-dienoyl-CoA isomerase, mitochondrial precursor (ECH1), hydroxyacylglutathione hydrolase, mitochondrial isoform 2 (HAGH)] were identified in $\geq 2$ protein spots.

Among GBC stage IIIA, three proteins (ALDOA, ALDOB and LTF) were identified in $\geq 3$ protein spots while one protein, catalase (CAT), was identified in $\geq 2$ protein spots (Supplementary Table S3). Detection of the same protein in multiple spots could be due to the existence of their isoforms, although we do not have any evidence for this.

\section{Clinical verification by dot blot assay}

Due to the presence of multiple proteins in a given gel spot, clinical verification was performed for two of the important proteins, ANXA1 and HSPD1, identified from protein spots showing immunoreactivity in early stage GBC cases (Supplementary Figures S2 and S3C). Clinical verification using individual blood plasma from 52 GBC cases (GBC stage I, II, III and IV) and 89 controls (healthy volunteers and GSD cases) (Table 1) showed significantly high autoantibody levels for ANXA1 in early stage GBC (stage I and II) as well as in GBC Stage IIIA ( $p$ value $<0.05$ ) (Fig. 3a, Table 2 ). However, no significant difference in the autoantibody level for HSPD1 was found in GBC (Fig. 3d). As we detected multiple proteins from the single immunoreactive spot, it is possible that HSPD1 was not contributing to immunoreactivity for the specific spot and thus there is a need to explore other proteins identified from the spot. Receiver operating characteristic (ROC) curve analysis of ANXA1 for early stage showed an Area under the ROC Curve (AUC) of 0.69 (95\% CI, 0.52-0.86), with 41.7\% sensitivity against a specificity of $89.9 \%$ in comparison to controls (Fig. 4a), while AUC was 0.5822 for the advanced stage with $15 \%$ sensitivity against a specificity of $89.9 \%$ in comparison to controls (Table 2).

\section{IHC analysis}

We performed IHC analysis to study the expression of ANXA1 in 14 controls (2 healthy liver donors and 12 GSD cases) and 43 GBC cases (16 early stage and 27 advanced stage GBC cases) and found 'high' expression levels in $72 \%$ of GBC cases. The expression of ANXA1 was found as 'high' in $62.5 \%$ of early stage and $77.7 \%$ of advance stage cases whereas all the controls showed 'low' expression levels (Fig. 5, Supplementary Table S4).

\section{Discussion}

Autoantibody levels in serum against TAAs have shown promise for early detection of cancer [17]. In this study, we have analysed the autoantibody response in GBC. For this purpose, we prepared tumor tissue lysate, as a source of TAAs, from GBC cases (Stage I, II and IIIA) and performed immunodepletion of HSA and IgG to enrich low abundant proteins and reduce non-specific reactivity due to anti-human IgG that was used as a secondary antibody in $1 \mathrm{D}$ and 2-D immunoblotting experiments. Immunodepleted tumor tissue proteins were resolved by 2-DE followed by 2-D immunoblot analysis using pooled plasma from controls (healthy volunteers or GSD cases) and GBC cases (stage I and II or IIIA) which led to the identification of 25 protein spots showing high or specific immunoreactivity in GBC. Mass spectrometric analysis (LC-MS/MS) of 8 protein spots that showed intense immunoreactivity in early stage GBC and GBC stage IIIA led to the identification of 27 proteins. A literature survey of the identified proteins was done for their expression and humoral response in GBC or other cancers, based on which we selected proteins for verifications using individual plasma samples.

We focused on proteins identified from four of the intense immunoreactive spots (spot no. 9-12) detected 


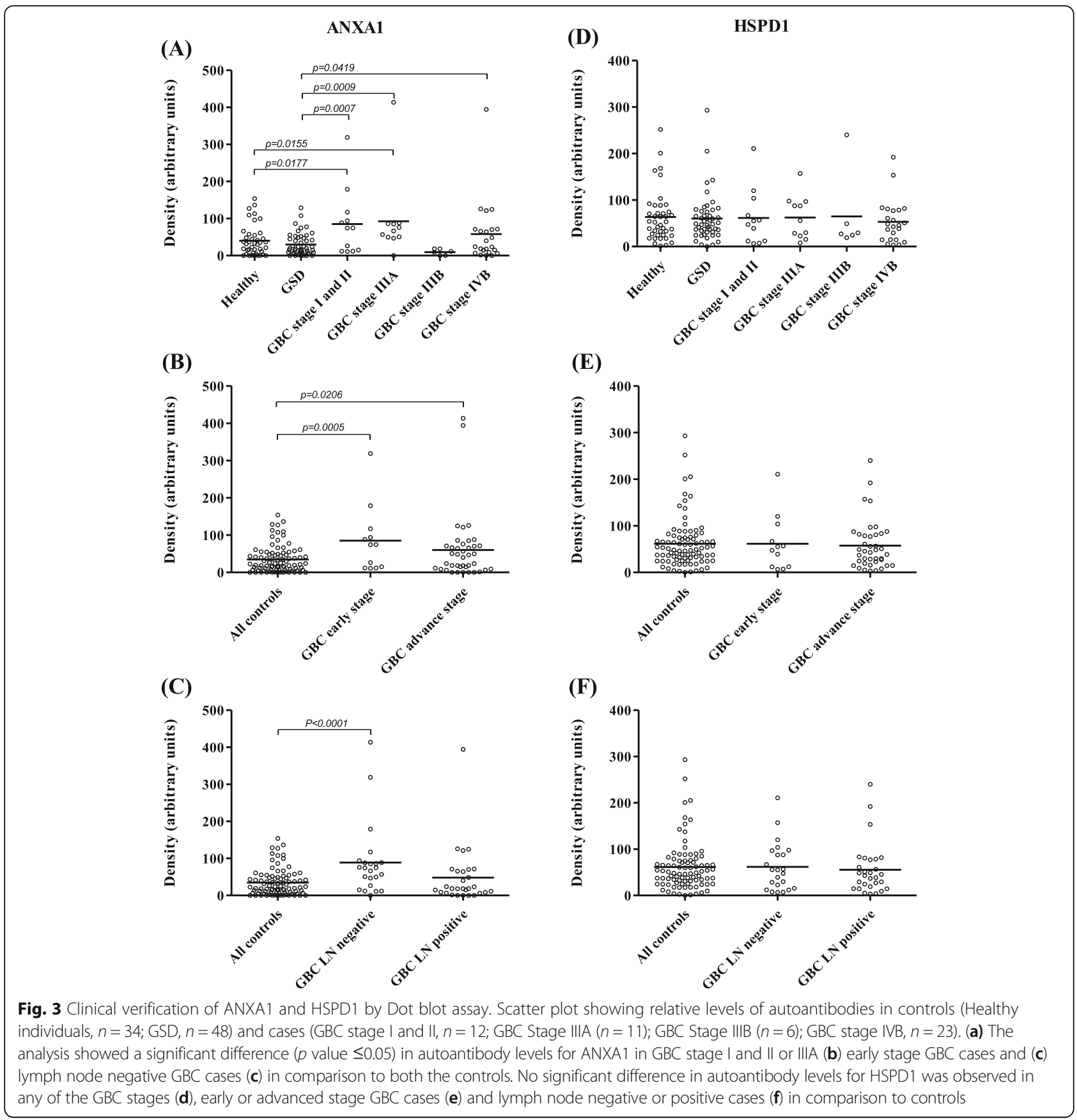

in early stage GBC. Out of 17 proteins identified from these four spots, increased autoantibody levels have been reported against seven of them including ANXA1, HSPD1, CA1, CA2, ALDOA, CTSD, and ECH1. One of these proteins, ANXA1, is previously reported to be overexpressed in GBC tissue in comparison to peri-tumoral tissue (PTs), adenomatous polyp and chronic cholecystitis $(\mathrm{CC})$. The level of ANXA1 was found to be significantly lower in early stage GBC than those in the advanced GBC cases [18]. ANXA1 is also reported to be overexpressed in CCA and could distinguish CCA from HCC [19]. ANXA1 is a calcium- and phospholipid-binding protein involved in cellular signal transduction pathways associated with inflammation, cell differentiation and cell proliferation [20]. Dysregulation and altered localization of ANXA1 have been correlated with tumor development and progression in several cancers including oral squamous cell carcinoma and gastric adenocarcinoma. It is also reported to promote tumor invasion and metastasis [21]. Autoantibody response against ANXA1 is previously reported in lung cancer and is one of the proteins among the panel of six TAAs (p53, NY-ESO1, Annexin I, CAGE, GBU4-5, 
Table 2 Statistical analysis of Dot blot data showing sensitivity or specificity for autoantibody levels for ANXA1

\begin{tabular}{|c|c|c|c|c|c|}
\hline Group comparison & $p$ value & Summary & AUC & Sensitivity (\%) & Specificity (\%) \\
\hline GBC stage I and II vs Healthy & 0.0177 & $*$ & 0.6545 & 25 & 92.68 \\
\hline GBC stage IIIA vs Healthy & 0.0155 & * & 0.7395 & 18.18 & 82.93 \\
\hline GBC stage IIIB vs Healthy & 0.0789 & ns & 0.7561 & 33.33 & 87.8 \\
\hline GBC stage IVB vs Healthy & 0.2635 & ns & 0.5483 & 17.39 & 92.68 \\
\hline GBC stage I and II vs GSD & 0.0007 & $* * *$ & 0.724 & 41.67 & 95.83 \\
\hline GBC stage IIIA vs GSD & 0.0009 & $* * *$ & 0.8011 & 45.45 & 91.67 \\
\hline GBC stage IIIB vs GSD & 0.1043 & ns & 0.7153 & 33.33 & 91.67 \\
\hline GBC stage IVB vs GSD & 0.0419 & * & 0.5951 & 17.39 & 91.67 \\
\hline GBC Early stage vs All controls & 0.0005 & $* * *$ & 0.6919 & 41.67 & 89.89 \\
\hline GBC Advance stage vs All controls & 0.0206 & * & 0.5822 & 15.00 & 89.89 \\
\hline LN negative vs All controls & $<0.0001$ & $* * *$ & 0.7306 & 30.43 & 89.89 \\
\hline LN positive vs All controls & 0.2102 & ns & 0.5099 & 13.79 & 89.89 \\
\hline All cases vs All controls & 0.0037 & $* *$ & 0.6075 & 15.38 & 95.51 \\
\hline
\end{tabular}

GBC Gallbladder carcinomas, GSD Gallstone disease, AUC area under curve, $C l$ confidence interval, ns not significant

and SOX2) that can detect up to $40 \%$ of all lung cancers in the disease groups, with a specificity of $90 \%[22,23]$. Linear peptide antigen from ANXA1 analyzed by enzyme-linked immunosorbent assay (ELISA) showed a significant increase in antibody levels in lung and breast cancer [24, 25].

In view of the above, we selected ANXA1 for clinical verifications and analyzed autoantibody levels against recombinant ANXA1 in early and advanced stage GBC cases using Dot blot assay. The results showed a significant increase in autoantibody levels against ANXA1 in early stage GBC (stage I and II) in comparison to controls (Fig. 3b, Table 2). Receiver operating characteristic (ROC) curve analysis for ANXA1 showed an Area under the ROC Curve (AUC) of 0.69 (95\% CI: 0.52-0.86), with $41.7 \%$ sensitivity against a specificity of $89.9 \%$ in comparison to controls (Fig. 4a). GBC stage I, II and IIIA are lymph node (LN) negative or non-metastatic stages where tumor cells are not spread to nearby lymph nodes. LN metastasis is the earliest sign of metastatic spread and is one of the established predictors of poor outcome in GBC patients [26]. We also compared the autoantibody levels for ANXA1 in LN negative (GBC stage I, II and IIIA) and LN positive (GBC stage IIIB, IVB) cases and found a significant increase of autoantibody levels in LN negative GBC cases. Receiver operating characteristic (ROC) curve analysis for ANXA1 showed an Area under the ROC Curve (AUC) of 0.73 (95\% CI: 0.61-0.85), with 30.4\% sensitivity against a specificity of $89.9 \%$ in comparison to controls (Fig. 4b). The LN positive GBC cases did not show any significant increase in autoantibody levels of ANXA1 (Fig. 3c, Table 2), possibly due to lower immunogenicity of cancer cells in LN metastatic cases $[27,28]$. The mechanism for the elevation of
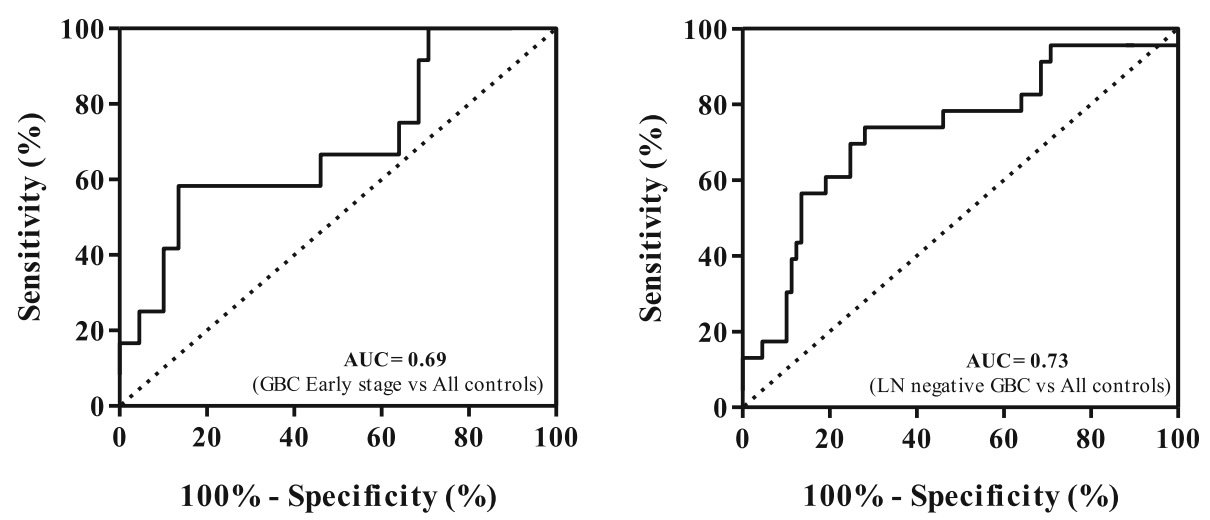

Fig. 4 Receiver operating characteristic (ROC) curve for ANXA1 antibodies in plasma from GBC cases and controls. (a) ROC curve analysis showed an Area under the ROC Curve (AUC) of 0.69 ( $95 \%$ Cl: $0.52-0.86$ ) with $41.7 \%$ sensitivity against a specificity of $89.9 \%$ in early stage GBC in comparison to controls and (b) an AUC of 0.73 (95\% Cl: $0.61-0.85$ ) with $30.4 \%$ sensitivity against a specificity of $89.9 \%$ in lymph node negative $\mathrm{GBC}$ in comparison to controls 


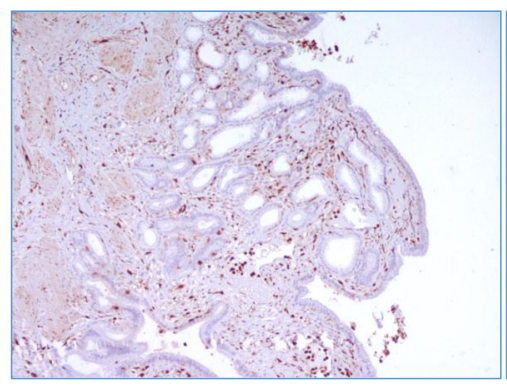

Healthy liver donor

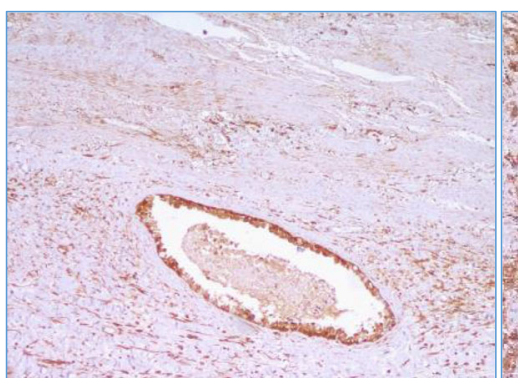

Early stage GBC

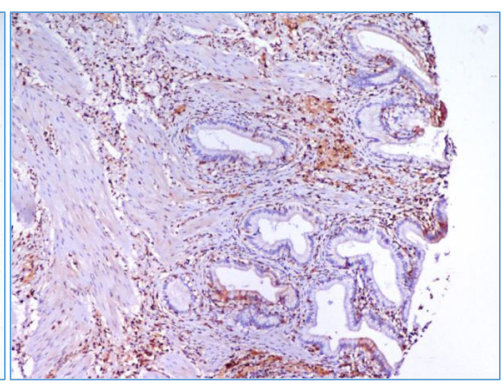

GSD

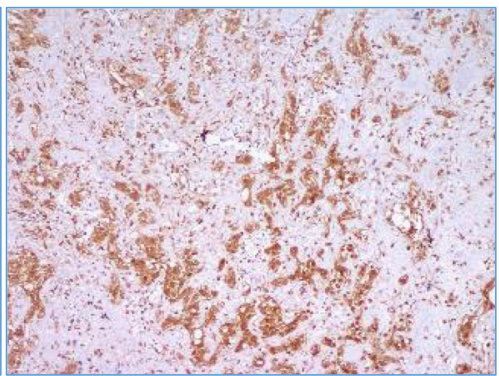

Advanced stage GBC

Fig. 5 Representative IHC images showing expression of ANXA1 in controls and cases. IHC analysis performed using FFPE tissue from 14 controls (2 Healthy liver donors and 12 GSD cases) and 43 GBC cases (16 early stage and 27 advanced stage GBC cases) showed 'high' expression levels in $72 \%$ of GBC cases. ANXA1 expression was 'high' in $77.7 \%$ of advanced stage and $62.5 \%$ of early stage GBC cases whereas all the controls showed 'low' expression levels. The details of tissue microarrays and individual tissue sections used and IHC procedure is described in the Methods and IHC scoring criteria are shown in Supplementary Table 54

IgG antibodies for ANXA1 is not clear. The autoantibody levels and clinical parameters like total leukocyte count (TLC), liver enzymes, cholestasis were compared (Supplementary Figure S1) and we did not find any correlation of the high or low autoantibody levels with any of the clinical parameters (Supplementary Table S5) suggesting that increased autoantibody levels in GBC patients were independent of these parameters and was possibly specific to cancer. There are independent reports on increased expression levels $[18,19]$ or increased autoantibodies for ANXA1 [22, 23] in several cancers, however, there is no report on a correlation between increased expression levels and autoantibody levels. In the present study, IHC analysis showed 'high' expression of ANXA1 in $72 \%$ GBC cases while all the controls showed 'low' expression levels suggesting that increased expression levels of ANXA1 may have a role in eliciting antibody response in GBC. In order to find if there is any correlation between ANXA1 expression and autoantibody levels, we compared the expression of ANXA1 in 16 GBC cases (8 with high autoantibody levels and 8 with low autoantibody levels against ANXA1). We did not find any correlation of ANXA1 expression and autoantibody levels suggesting that there may be other factors such as post-translational modification or altered localization in tumor cells, for increased autoantibody levels against ANXA1 in GBC.

On similar lines, we also selected another protein HSPD1 for verification. HSPD1 is a chaperonin, involved in regulating apoptosis in cancer. An increased autoantibody level against HSPD1 has been reported in $\mathrm{HCC}$ and breast cancer and proposed as a marker for early detection [29-31]. However, our results did not show any significant difference in autoantibody levels in GBC (Fig. 3e and f), thus enhancing the importance of the specific observation made with ANXA1 and the need to investigate other individual proteins detected in the same spot.

The sample size of early stage GBC cases used for the study was small and the analysis could be expanded with a larger cohort of clinical samples for higher confidence in the results. Also, other individual proteins identified from the immunoreactive protein spots such as CA1, CA2, ALDOA may be analyzed for the autoantibody levels in GBC cases to expand the panel of proteins with improved sensitivity to detect $G B C$ at early stage.

\section{Conclusions}

This study involves the application of SERPA approach for the detection of protein spots with high or specific 
immunoreactivity in early stage GBC. Our data on clinical verification revealed significantly high autoantibody levels against ANXA1 in early stage GBC in comparison to controls suggesting its potential for early detection of GBC. The analysis may be carried out further in a larger cohort of samples for clinical applications.

\section{Supplementary Information}

The online version contains supplementary material available at https://doi. org/10.1186/s12885-020-07676-6.

Additional file 1: Supplementary Figure S1. Scatter plot showing variations in TLC, liver enzymes, bilirubin and cholestasis among the cases and controls used for the study. (A) TLC (B) Bilirubin (C) SGOT (D) SGPT (E) ALP levels in samples used for pooling of plasma or tissue for the discovery phase and individual plasma samples for all GSD and GBC cases used for verification study. The dotted line represents the normal levels of these parameters. The solid line represents the bilirubin levels > $2 \mathrm{mg} / \mathrm{dL}$ suggests cholestasis. The data was available for $\sim 68 \%$ of the samples i.e. 33 out of 48 GSD cases and 35 out of 52 GBC cases used for the study and was used for analysis. Normal levels for TLC- 4000-10,000 per $\mathrm{mm}^{3}$, Bilirubin- 0.3-1.2 mg/dL, SGOT- > $35 \mathrm{U} / \mathrm{L}$, SGPT- > $35 \mathrm{U} / \mathrm{L}$ and ALP- 30-120 U/L. TLC- Total leukocyte count, SGOT-Serum Glutamic Oxaloacetic Transaminase or Aspartate transaminase, SGPT- Serum glutamic pyruvic transaminase or alanine aminotransferase, ALP- Alkaline phosphatase, GSD- Gallstone disease, GBC- Gallbladder cancer.

Additional file 2: Supplementary Figure S2. Dot blot images showing autoantibody levels of ANXA1 and HSPD1 in cases and control groups. For Dot blot assay, a total of 75 ng recombinant protein was spotted on PVDF membrane followed by blocking and incubation with individual blood plasma samples from 82 controls (healthy individuals and GSD) and 52 cases (early and advanced stage GBC) overnight at $4{ }^{\circ} \mathrm{C}$. The blots were then incubated with secondary antibody (anti-human IgG HRP conjugated) followed by development using SuperSignal ${ }^{\mathrm{TM}}$ West Femto Maximum Sensitivity Substrate (Thermo). The images were acquired using Chemidoc MP (Bio-Rad, USA) and densitometric analysis was performed using ImageLab software version 4.1 (Bio-Rad, USA). The relative levels of autoantibodies in controls and cases are represented as scatter plot in Fig. 3. H: Healthy; GSD: Gallstone disease; I + II: GBC stage I and II; IIIA: GBC stage IIIA; IIIB: GBC stage IIIB; IVB: GBC Stage IVB. The fulllength blot images are presented in Supplementary Figure \$3.

Additional file 3: Supplementary Figure S3. The full-length blot images of Fig. 2 and Fig. S2. (A) The full-length blot images of Fig. 2a. The cropped image includes the lanes ('Healthy', 'GSD', 'GBC stage I and II', 'GBC stage IIIA' and 'Negative') from full-length blot image with 'high exposure'. The lane with MW marker is from the blot image with 'low exposure'. All these blots were developed together. The cropping of the image is indicated with red dashed line (B) The full-length blot images of Fig. 2c. Immunodepleted tumor tissue proteins were resolved by 2-DE and electro transferred onto PVDF membrane. The blots were separately incubated with pooled plasma from healthy individuals or GSD cases or GBC Stage I and II or GBC stage IIIA cases. The blots were first incubated with anti-human lgG conjugated with HRP (i), developed using ECL kit and image was acquired. The same blot was subsequently developed after incubation with StrepTactin-HRP Conjugate (ii) to visualize the MW marker. The full-length 2-D blot image (i) and the lane with MW marker (ii) was cropped and aligned appropriately for inclusion in the Fig. 2C. The cropping of the blot images is indicated with red dashed line. (C) The full-length Dot blot images of Fig. S2. The Dot blot assay for 52 cases and 89 controls was performed in 4 independent sets (Set 1-4). Two recombinant proteins, HSPD1 and ANXA1, were spotted on the same PVDF membrane and exposed to individual plasma samples. The reactive spot areas on each dot blot were quantified as described under Methods and the spots cropped to align the data for HSPD1 and ANXA1 separately as shown in Fig. S2.

Additional file 4: Supplementary Table S1. List of identified proteins from immunoreactive protein spots by mass spectrometric analysis.
Additional file 5: Supplementary Table S2. List of proteins and peptides identified by mass spectrometric analysis from immunoreactive protein spots.

Additional file 6: Supplementary Table S3. Molecular functions of proteins identified by mass spectrometric analysis. Molecular functions are derived from HPRD database [http://www.hprd.org; Ref. [16]] and uniprot database [www.uniprot.org/uniprot].

Additional file 7: Supplementary Table S4. Expression of ANXA1 in control and GBC tissue using $I H C$ analysis. IHC was performed on formalin-fixed paraffin-embedded (FFPE) tissue microarrays (TMAs) and individual tissue sections. Two in-house TMA blocks were constructed using the FFPE blocks and included 14 controls ( 2 healthy liver donors and 12 GSD cases) and 31 GBC cases (9 early stage and 22 advanced stage). Each TMA block consisted of 22 cores of $2 \mathrm{~mm}$ diameter and $4 \mu \mathrm{m}$ sections were cut from the TMA block for carrying out IHC. Individual tissue sections (FFPE) of GBC (7 early stage and 5 advanced stage) were also for $\mathrm{IHC}$ analysis. The staining intensity of cancer cells was scored as $0,1+$, $2+/ 3+$ indicating negative, low, and strong staining, respectively. All the cases showed $2+/ 3+$ staining intensity. The distribution of staining of cancer cells was scored as 0 (less than 10\% of cells staining), $1+(10-<$ $25 \%$ of cell staining), $2+(25-<50 \%$ of cells staining) and $3+(\geq 50 \%$ of cells staining). ANXA1 expression was considered 'high' if the percentage distribution was $\geq 25 \%$ and 'low' if it was $<25 \%$. IHC data analysis was done by two independent pathologists.

Additional file 8: Supplementary Table S5. Correlation of the autoantibody levels with clinical parameters TLC, Bilirubin, SGOT, SGPT, ALP levels in GBC cases. We did not find any correlation of increased autoantibody levels with increased levels of TLC, liver enzymes. The values in bold are above the normal range.

\section{Abbreviations}

GBC: Gallbladder carcinoma; GSD: Gallstone disease;

CCA: Cholangiocarcinomas; TAAs: Tumor-associated antigens; LN: Lymph node; SERPA: Serological proteome analysis; 2-DE: Two-dimensional gel electrophoresis; LC-MS/MS: Liquid chromatography-tandem mass spectrometry; HCD: Higher-energy collision dissociation; FDR: False discovery rate; ANXA1: Annexin A1; HSPD1: 60 kDa Heat shock protein, mitochondrial

\section{Acknowledgements}

We acknowledge Geeta Chauhan, Laboratory Technician at GIPMER, New Delhi, who was involved in clinical specimen collection, coordination and clinical documentation, and Ankit P. Jain for mass spectrometric analysis carried out at the MS facility at the Institute of Bioinformatics, Bangalore.

\section{Authors' contributions}

PG, PS, RS were involved in study design and research; JA, RP, PG, RVP were involved in data acquisition, analysis and interpretation of data; JA, RP were involved in statistical analysis; PS, AA, JA, RP, VJ were involved in characterization and patient samples; PS, SG, PG, JA were involved in $\mathrm{HC}$ data analysis; PG, JA and RVP were involved in manuscript writing; RS, AA, SK had critically reviewed the manuscript; PG and PS were involved in study supervision. All authors read and approved the final manuscript.

\section{Funding}

The work reported here was financially supported by the Department of Science and Technology (DST)- Science and Engineering Research Board (SERB) (Project ID- YSS/2015/001321), Govt. of India, New Delhi and Indian Council of Medical Research (ICMR) (Project ID- 2014-1182), Govt. of India, New Delhi. Javed Akhtar is a Ph.D. student registered at Jamia Hamdard, New Delhi and a recipient of Senior Research Fellowship (SRF) from the Council of Scientific and Industrial Research (CSIR), Govt. of India. Ratna Priya is a Ph.D. student registered at Jamia Hamdard, New Delhi and DSTInnovation in Science Pursuit for Inspired Research (INSPIRE)- Senior Research Fellow (SRF). Vaishali Jain is a recipient of Junior Research Fellowship (JRF) from the Council of Scientific and Industrial Research (CSIR), Govt. of India.

\section{Availability of data and materials}

All data generated or analysed during this study are included in this published article and its supplementary information files. 


\section{Ethics approval and consent to participate}

Clinical samples from patients visiting GIPMER, Delhi, were collected for the study after approval from the Institutional Human Ethics Committee [F.1/IEC/ MAMC/(52/1/2016/No. 36) and NIP-IEC/21-12/05]. All patients provided informed consent to participate in the study and written informed consent was obtained.

\section{Consent for publication}

Not applicable.

\section{Competing interests}

The authors declare that they have no competing interests.

\section{Author details}

'Laboratory of Molecular Oncology, ICMR- National Institute of Pathology, Safdarjung Hospital Campus, New Delhi 110029, India. ${ }^{2}$ Jamia HamdardInstitute of Molecular Medicine, Jamia Hamdard, New Delhi 110062, India. ${ }^{3}$ Manipal Academy of Higher Education (MAHE), 576104 Manipal, India. ${ }^{4}$ Govind Ballabh Pant Institute of Postgraduate Medical Education and Research (GIPMER), New Delhi 110002, India. ${ }^{5}$ Department of Biochemistry, Sri Venkateswara College, University of Delhi, New Delhi 110021, India.

${ }^{6}$ Institute of Bioinformatics, International Tech Park, Bangalore 560066, India.

Received: 28 February 2020 Accepted: 22 November 2020

Published online: 01 December 2020

\section{References}

1. Mahdavifar N, Pakzad R, Ghoncheh M, Gandomani HS, Salehiniya H. Epidemiology, incidence, and mortality of gallbladder cancer and its relation with development in the world. Ann Trop Med Public Health. 2017:10:563-70.

2. Malhotra RK, Manoharan N, Shukla NK, Rath GK. Gallbladder cancer incidence in Delhi urban: a 25-year trend analysis. Indian J Cancer. 2017; 54(4):673-7.

3. Randi G, Franceschi S, La Vecchi C. Gallbladder cancer worldwide: geographical distribution and risk factors. Int J Cancer. 2006;118:1591-9.

4. Zhu AX, Hong TS, Hezel AF, Kooby DA. Current management of gallbladder carcinoma. Oncologist. 2010;15(2):168-81.

5. Shah SH, Gupta N, Gupta G, Mehta A, Singh S. Lymph node micrometastasis in gallbladder cancer. Indian J Gastroenterol. 2017;36(4):268-74.

6. Tan HT, Low J, Lim SG, Chung MC. Serum autoantibodies as biomarkers for early cancer detection. FEBS J. 2009:276:6880-904.

7. Casiano CA, Mediavilla-Varela M, Tan EM. Tumor-associated antigen arrays for the serological diagnosis of cancer. Mol Cell Proteomics. 2006:5:1745-59.

8. Macdonald IK, Parsy-Kowalska CB, Chapman CJ. Autoantibodies: opportunities for early Cancer detection. Trends Cancer. 2017:3(3):198-213.

9. Desmetz C, Maudelonde T, Mangé A, Solassol J. Identifying autoantibody signatures in cancer: a promising challenge. Expert Rev Proteomics. 2009; 6(4):377-86.

10. Shukla S, Govekar RB, Sirdeshmukh R, Sundaram CS, D'Cruz AK, Pathak KA, et al. Tumor antigens eliciting autoantibody response in cancer of gingivobuccal complex. Proteomics Clin Appl. 2007;1(12):1592-604.

11. Looi KS, Nakayasu ES, Diaz RA, Tan EM, Almeida IC, Zhang JY. Using proteomic approach to identify tumor-associated antigens as markers in hepatocellular carcinoma. J Proteome Res. 2008;7(9):4004-12.

12. Gupta P, Suman S, Mishra M, Mishra S, Srivastava N, Kumar V, et al. Autoantibodies against TYMS and PDLIM1 proteins detected as circulatory signatures in Indian breast cancer patients. Proteomics Clin Appl. 2016;10(5): 564-73.

13. Mustafa MZ, Nguyen VH, Le Naour F, De Martin E, Beleoken E, Guettier C, et al. Autoantibody signatures defined by serological proteome analysis in sera from patients with cholangiocarcinoma. J Transl Med. 2016;14:17.

14. Amin MB, Greene FL, Edge SB, Compton CC, Gershenwald JE, Brookland RK, et al. The eighth edition AJCC Cancer staging manual: continuing to build a bridge from a population-based to a more "personalized" approach to cancer staging. CA Cancer J Clin. 2017:67(2):93-9.

15. Görg A, Postel W, Günther S, Friedrich C. Horizontal twodimensional electrophoresis with immobilized $\mathrm{pH}$ gradients using PhastSystem. Electrophoresis. 1988;9(1):57-9.
16. Keshava Prasad TS, Goel R, Kandasamy K, Keerthikumar S, Kumar S, Mathivanan S, et al. Human protein reference database--2009 update. Nucleic Acids Res. 2009;37:D767-72.

17. Zaenker P, Gray ES, Ziman MR. Autoantibody production in Cancer--the Humoral immune response toward autologous antigens in Cancer patients. Autoimmun Rev. 2016;15(5):477-83.

18. Yang LP, Yang ZL, Tan XG, Miao XY. Expression of annexin A1 (ANXA1) and A2 (ANXA2) and its significance in benign and malignant lesions of gallbladder. ZhonghuaZhong Liu ZaZhi. 2010;32(8):595-9.

19. Hongsrichan N, Rucksaken R, Chamgramol Y, Pinlaor P, Techasen A, Yongvanit $\mathrm{P}$, et al. Annexin A1: a new immunohistological marker of cholangiocarcinoma. World J Gastroenterol. 2013;19(16):2456-65.

20. Mussunoor S, Murray Gl. The role of annexins in tumour development and progression. J Pathol. 2008;216(2):131-40.

21. Sheikh MH, Solito E, et al. Int J Mol Sci. 2018;19(4):pii: E1045.

22. Boyle P, Chapman CJ, Holdenrieder S, Murray A, Robertson C, Wood WC, et al. Clinical validation of an autoantibody test for lung cancer. Ann Oncol. 2011;22(2):383-9.

23. Murray A, Chapman CJ, Healey G, Peek L, Parsons G, Baldwin D, et al. Technical validation of an autoantibody test for lung cancer. Ann Oncol. 2010;21(8):1687-93.

24. Wang W, Guan S, Sun S, Jin Y, Lee KH, Chen Y, et al. Detection of circulating antibodies to linear peptide antigens derived from ANXA1 and DDX53 in lung cancer. Tumour Biol. 2014;35(5):4901-5.

25. Huang Y, Zhang C, Chen C, Sun S, Zheng H, Wan S, et al. Investigation of circulating antibodies to ANXA1 in breast cancer. Tumour Biol. 2015;36(2): 1233-6.

26. Shirai Y, Sakata J, Wakai T, Ohashi T, Ajioka Y, Hatakeyama K. Assessment of lymph node status in gallbladder cancer: location, number, or ratio of positive nodes. World J SurgOncol. 2012;10:87.

27. Shih NY, Lai HL, Chang GC, Lin HC, Wu YC, Liu JM, et al. Anti-alpha-enolase autoantibodies are down-regulated in advanced cancer patients. Jpn J Clin Oncol. 2010:40(7):663-9.

28. Ye L, Wang Y, Liu T, Liu B, Liu F, Liu B. Circulating antibody to ANXA1 may be a potential biomarker for early diagnosis of esophageal cancer. Int J Clin Exp Pathol. 2017;10(2):2284-9.

29. Hong Y, Long J, Li H, Chen S, Liu Q, Zhang B, et al. An analysis of Immunoreactive signatures in early stage hepatocellular carcinoma. EBioMedicine. 2015;2(5):438-46.

30. Yang Z, Chevolot Y, Géhin T, Solassol J, Mange A, Souteyrand E, et al. Improvement of protein immobilization for the elaboration of tumorassociated antigen microarrays: application to the sensitive and specific detection of tumor markers from breast cancer sera. BiosensBioelectron. 2013:40(1):385-92

31. Xia J, Shi J, Wang P, Song C, Wang K, Zhang J, et al. Tumour-associated autoantibodies as diagnostic biomarkers for breast Cancer: a systematic review and meta-analysis. Scand J Immunol. 2016;83(6):393-408.

\section{Publisher's Note}

Springer Nature remains neutral with regard to jurisdictional claims in published maps and institutional affiliations.

Ready to submit your research? Choose BMC and benefit from

- fast, convenient online submission

- thorough peer review by experienced researchers in your field

- rapid publication on acceptance

- support for research data, including large and complex data types

- gold Open Access which fosters wider collaboration and increased citations

- maximum visibility for your research: over $100 \mathrm{M}$ website views per year

At $\mathrm{BMC}$, research is always in progress.

Learn more biomedcentral.com/submission 